\title{
Airway abnormalities in Jarcho-Levin syndrome: a report of two cases
}

\author{
Marjorie Schulman, Marco T Gonzalez, Michael R Bye
}

\begin{abstract}
Two infants with the Jarcho-Levin syndrome of vertebral anomalies underwent flexible fibre optic bronchoscopy. Central airway abnormalities not amenable to surgical correction were found in both patients. These abnormalities may contribute significantly to the respiratory failure seen in affected infants, and should be considered when evaluating continuing medical support.

( $\mathcal{F}$ Med Genet 1993;30:875-6)
\end{abstract}

The Jarcho-Levin syndrome of vertebral anomalies, variously termed costovertebral dysplasia, spondylothoracic dysostosis, and occipito-facial-cervico-thoracic-abdomino-digital dysplasia, is characterised by malformations of the thoracic vertebrae and ribs. ${ }^{1-3}$ Respiratory compromise resulting from a small thoracic cavity is associated with a very high mortality. Over 60 cases have been reported to date, primarily in children of Puerto Rican origin. ${ }^{4}$ Inheritance is most commonly autosomal recessive, but variants have been reported showing an autosomal dominant pattern. ${ }^{5}$

A plethora of non-skeletal abnormalities has been described in Jarcho-Levin syndrome, including neural tube defects, Arnold-Chiari malformation, and renal and urinary tract abnormalities. ${ }^{6-8}$ We report two patients with Jarcho-Levin syndrome who underwent flexible fibre optic bronchoscopy, showing heretofore unreported anomalies of the central airway.

\section{Case reports}

CASE 1

Case 1 was a $2740 \mathrm{~g}$ term female born by elective repeat caesarean section to non-consanguineous Puerto Rican parents. The mother was a 33 year old gravida 5 , para 1 , abortions (elective) 3 with a history of valvular heart disease, gestational diabetes, and intravenous heroin use eight years before this pregnancy. Her 4 year old daughter, fathered by a previous husband, is well. This pregnancy was complicated by polyhydramnios. Apgar scores were 7 at one minute and 8 at five minutes. The diagnosis of Jarcho-Levin syndrome was made on the day of birth. The infant had respiratory distress requiring intubation in the delivery room. Physical examination showed marked kyphoscoliosis, a barrel chest, and a short trunk and neck. A chest radiograph (fig 1) showed a small thoracic cavity, a crab-like appearance of the rib cage, and multiple hemivertebrae. The infant tolerated extubation briefly, but developed cyanosis and respiratory

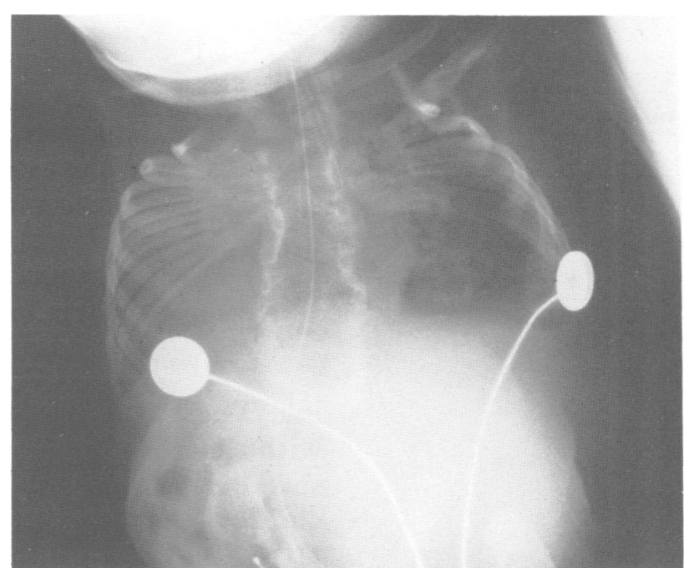

Figure 1 Chest radiograph of case 1.

acidosis and remained ventilator dependent thereafter. Additional studies included ultrasound examinations of the head and kidneys, neither of which indicated any abnormality.

Wheezing was noted during the first few days of life, which was unresponsive to aerosolised bronchodilators. Flexible fibre optic bronchoscopy was performed on day 5 of life. Right choanal stenosis was found. The cartilaginous rings could not be seen bulging through the tracheal lining. The carina was splayed and both mainstem bronchi were narrow. The infant's respiratory status deteriorated inexorably over several weeks. On day 47, mechanical ventilation was withdrawn at the family's request, in consultation with the institutional bioethics committee. She died within minutes of extubation. Necropsy was refused by the family.

CASE 2

Case 2, a male, was born at term to healthy, non-consanguineous Puerto Rican parents. The mother was a 30 year old primigravida. Her family history was unremarkable and the father was unavailable. Delivery was by caesarean section because of failure to progress in labour. The birth weight was $2900 \mathrm{~g}$. The diagnosis of Jarcho-Levin syndrome was made on the day of birth (fig 2). The infant never required mechanical ventilation, but required supplemental oxygen beginning at 3 months of age. Marked wheezing developed in early infancy, and the patient required admission to hospital numerous times for reactive airway disease. He underwent flexible fibre optic bronchoscopy at 19 months of age. The trachea was short and approximately one third of the way down made a sharp turn to the left. In the distal trachea, the cartilaginous rings could not be seen bulging through the tracheal lin-
NY 10461, USA

Received 26 February 1993. Revised version
13 April 1993. 


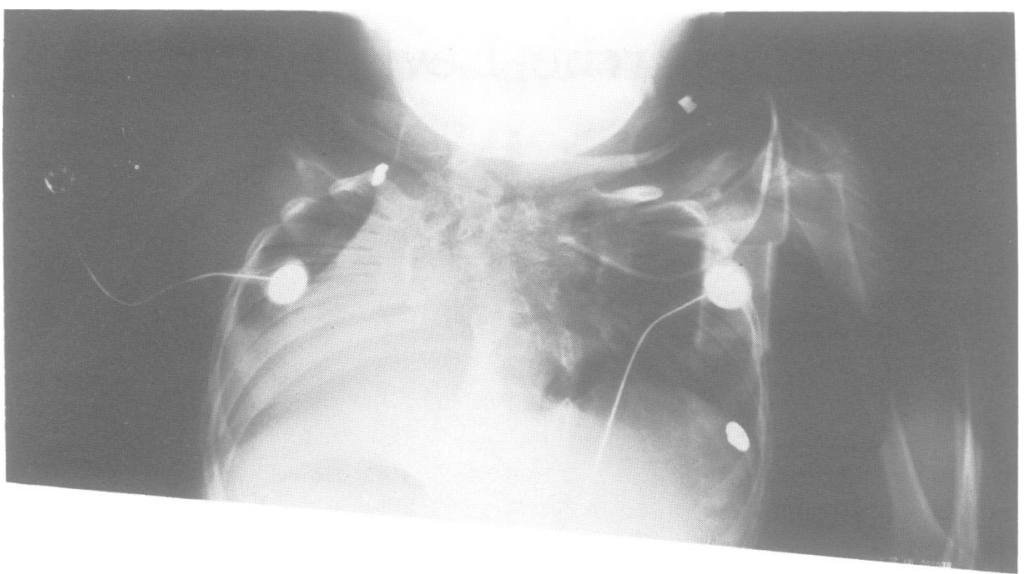

Figure 2 Chest radiograph of case 2.

ing. There was marked splaying of the carina with narrowing of the mainstem bronchi, severe enough to prevent insertion of the bronchoscope (Olympus Model BF-3C10, New Hyde Park, NY; outer diameter c $3.6 \mathrm{~mm}$ ). The patient developed chronic airflow obstruction over a period of several years, with baseline wheeze, chronic hypoxaemia, and carbon dioxide retention, and recurrent episodes of reactive airway disease. At the age of 5 , he is maintained on supplemental oxygen and several bronchodilators. He has hyperopia, astigmatism, bilateral inguinal hernias, multiple dental caries, and abscesses. Developmental evaluation showed minor motor delays, expressive language and perceptuomotor delays, and a Bayley Mental Development Index (MDI) of 83 , possibly related to recurrent hypoxaemia.

\section{Discussion}

These two patients with the Jarcho-Levin syndrome show abnormalities of the central airways which are, to the best of our knowledge, previously unreported. In both children, the cartilaginous rings normally seen bulging through the tracheal epithelium were obscured. This could be a manifestation of abnormality of the rings or dysplastic changes in the epithelium, either primary or secondary to recurrent aspiration. Both patients also had marked splaying of the carina and narrowing of the mainstem bronchi. In neither case were the anomalies amenable to surgical interven- tion such as tracheostomy or tracheal reconstruction.

Previous reports of this syndrome correlate the ability of affected subjects to survive with their degree of pulmonary insufficiency. ${ }^{9}$ Some authors have attempted to classify the syndrome into lethal and non-lethal forms, but such classification is not universally accepted. ${ }^{59}$ The inability of the infant described in case 1 to survive was presumably related to increased central airway resistance as well as pulmonary hypoplasia, resulting in hypoventilation and severe hypoxaemia. The continuing respiratory problems of case 2 , who is still alive, are related to his airway anomalies as well as his thoracic skeletal dysplasia.

The advisability of aggressive medical intervention in a newborn with Jarcho-Levin syndrome may be questioned. Earlier reports of the Jarcho-Levin syndrome contained an extremely poor prognosis for survival. ${ }^{23}$ However, more recent reviews report patients surviving many years, ${ }^{59}$ no doubt as a result of the improvement in acute and long term ventilatory management of newborns since the syndrome was first described. The ability of such infants to survive is principally related to the pulmonary consequences of their thoracic skeletal anomalies. These two cases show that central airway anomalies may contribute to the respiratory insufficiency. We do not feel that bronchoscopy is necessarily clinically beneficial in patients with this condition. However, the airway findings described above should be taken into account when counselling families of critically ill patients with Jarcho-Levin about the benefits of continuing intensive support.

1 Jarcho S, Levin PM. Hereditary malformations of the vertebral bodies. Fohns Hopkins Med f 1938;62:216-26.

2 Pérez-Comas A, Garcia-Castro JM. Occipito-facial-cervicothoracic-abdomino-digital dysplasia: Jarcho-Levin synthoracic-abdomino-digital dysplasia: Jarcho-Levin syn-

3 drome of vertebral anomalies. F Pediatr 1974;85:388-91. Philadelphia: Saunders, 1982:448.

Philadelphia: Saunders, 1982:448.
Romeo MG, Distefano G, DiBella D, et al. Familial Jarcho-

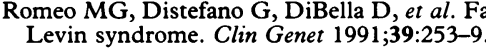

5 Karnes PS, Day D, Berry SA, Pierpont ME. Jarcho-Levin syndrome: four new cases and classification of subtypes. Am $\mathcal{F}$ Med Genet 1991;40:264-70.

6 Poor MA, Albert O, Griscom NT, Driscoll SG, Holmes LB Nonskeletal malformations in one of three siblings with Jarcho-Levin syndrome of vertebral anomalies. $\mathcal{f}$ Pediatr 1983;103:270-2.

7 Reyes MG, Morales A, Harris V, Barreta TM, Goldbard H Neural defects in Jarcho-Levin syndrome. $\mathcal{f}$ Child Neurol 1989;4:51-4.

8 Young S, Reyes MG, Uzoaru IL, Harris V. Arnold-Chiari malformation in Jarcho-Levin syndrome. $\mathcal{F}$ Child Neurol malformation in

9 Herold HZ, Edlitz M, Baruchin A. Spondylothoracic dysplasia: a report of ten cases with follow-up. Spine 1988; 13:478-81. 